Supplement of Clim. Past, 14, 601-608, 2018

https://doi.org/10.5194/cp-14-601-2018-supplement

(c) Author(s) 2018. This work is distributed under

the Creative Commons Attribution 4.0 License.

(c) (1)

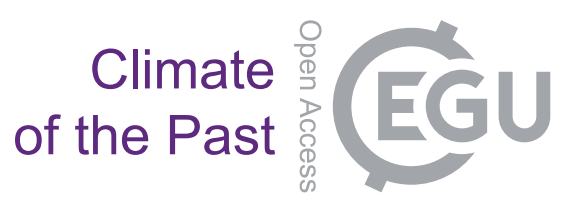

Supplement of

\title{
Particle shape accounts for instrumental discrepancy in ice core dust size distributions
}

Marius Folden Simonsen et al.

Correspondence to: Marius Folden Simonsen (msimonse@ fys.ku.dk)

The copyright of individual parts of the supplement might differ from the CC BY 4.0 License. 


\section{A Bags measured by the Coulter Counter}

A.1 Holocene

\begin{tabular}{|c|c|c|c|c|}
\hline $\begin{array}{l}\mathrm{Bag} \\
\mathrm{Bag}\end{array}$ & $\begin{array}{r}\text { Top } \\
\text { depth }(\mathrm{m})\end{array}$ & $\begin{array}{r}\text { Bottom } \\
\text { depth }(\mathrm{m})\end{array}$ & $\begin{array}{r}\text { Top age } \\
(\text { years b2k) }\end{array}$ & $\begin{array}{c}\text { Bottom age } \\
\text { (years b2k) }\end{array}$ \\
\hline 300 & 164.45 & 165.00 & 356 & 358 \\
\hline 304 & 166.65 & 167.20 & 363 & 365 \\
\hline 305 & 167.20 & 167.75 & 365 & 367 \\
\hline 321 & 176.00 & 176.55 & 395 & 397 \\
\hline 335 & 183.70 & 184.25 & 423 & 425 \\
\hline 714 & 392.15 & 392.70 & 1980 & 1989 \\
\hline 716 & 393.25 & 393.80 & 1998 & 2006 \\
\hline 746 & 409.75 & 410.30 & 2282 & 2293 \\
\hline 747 & 410.30 & 410.85 & 2293 & 2303 \\
\hline 755 & 414.70 & 415.25 & 2379 & 2390 \\
\hline 759 & 416.90 & 417.45 & 2423 & 2434 \\
\hline 760 & 417.45 & 418.00 & 2434 & 2446 \\
\hline 762 & 418.55 & 419.10 & 2457 & 2469 \\
\hline 788 & 432.85 & 433.40 & 2785 & 2799 \\
\hline 795 & 436.70 & 437.25 & 2884 & 2899 \\
\hline 797 & 437.80 & 438.35 & 2914 & 2928 \\
\hline 799 & 438.90 & 439.45 & 2943 & 2958 \\
\hline 800 & 439.45 & 440.00 & 2958 & 2974 \\
\hline 836 & 459.25 & 459.80 & 3588 & 3608 \\
\hline 837 & 459.80 & 460.35 & 3608 & 3629 \\
\hline 838 & 460.35 & 460.90 & 3629 & 3650 \\
\hline 840 & 461.45 & 462.00 & 3671 & 3692 \\
\hline 842 & 462.55 & 463.10 & 3713 & 3734 \\
\hline 847 & 465.30 & 465.85 & 3822 & 3845 \\
\hline 848 & 465.85 & 466.40 & 3845 & 3868 \\
\hline 850 & 466.95 & 467.50 & 3890 & 3914 \\
\hline 851 & 467.50 & 468.05 & 3914 & 3937 \\
\hline 852 & 468.05 & 468.60 & 3937 & 3961 \\
\hline 854 & 469.15 & 469.70 & 3984 & 4008 \\
\hline
\end{tabular}

A.2 Glacial

\begin{tabular}{lrrrr}
\hline $\begin{array}{l}\text { Bag } \\
\text { Bag }\end{array}$ & $\begin{array}{r}\text { Top } \\
\text { depth }(\mathrm{m})\end{array}$ & $\begin{array}{r}\text { Bottom } \\
\text { depth }(\mathrm{m})\end{array}$ & $\begin{array}{r}\text { Top age } \\
(\text { years b2k) }\end{array}$ & $\begin{array}{r}\text { Bottom age } \\
\text { (years b2k) }\end{array}$ \\
\hline 972 & 534.05 & 534.60 & 17760 & 21170 \\
973 & 534.60 & 535.15 & 21170 & 24745 \\
974 & 535.15 & 535.70 & 24745 & 28783 \\
975 & 535.70 & 536.25 & 28783 & 33885 \\
\hline
\end{tabular}

\section{B Coincidence}

There is no indication of coincidence in the Abakus. If two small particles coincide in the Abakus detector, their combined shadow will make them look like a larger particle. This effect might skew the Abakus distribution towards showing 


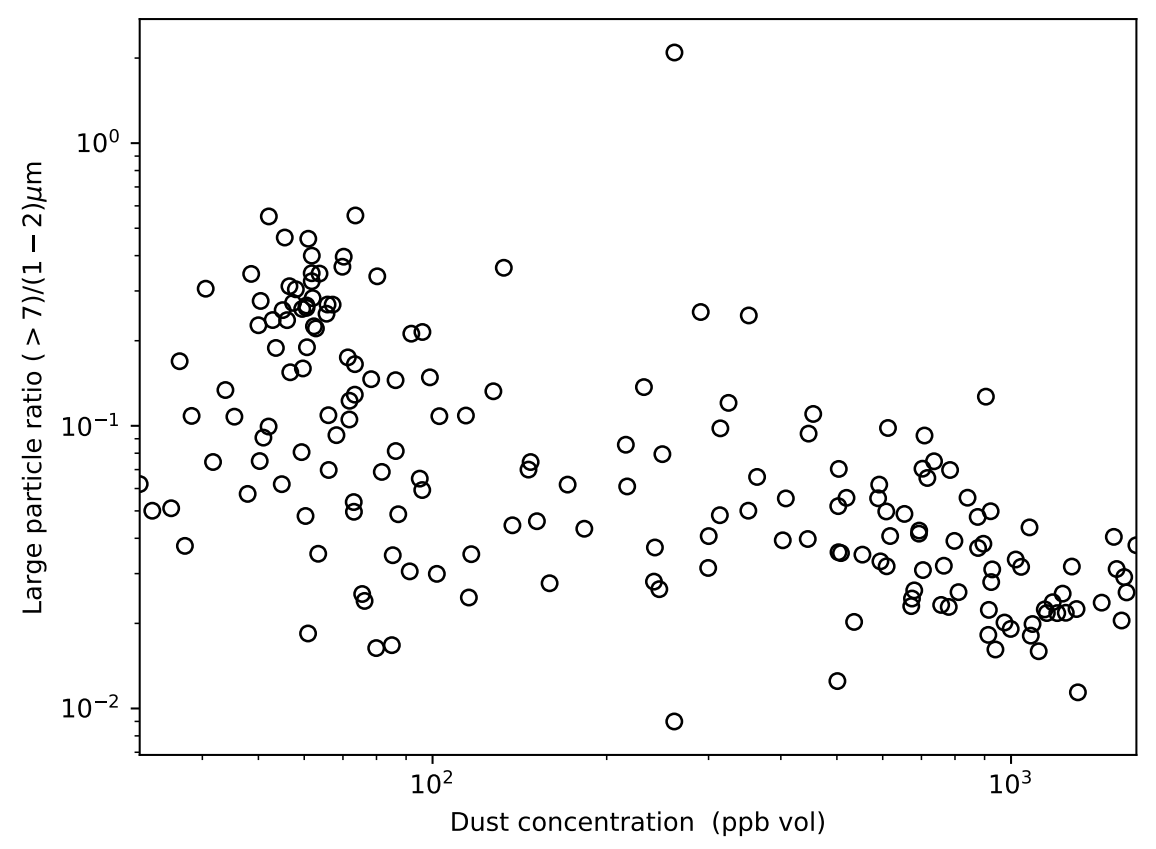

Figure A: The ratio between small and large particle concentration by volume as a function of total dust concentration. The points are $10 \mathrm{~cm}$ sections from 533-554 m depth.

more large particles. It has previously been shown that the coincidence effect is negligible for concentrations below 240,000 particles/mL (Saey, 1998). The highest dust concentrations measured in the RECAP core were around 220,000 particles $/ \mathrm{mL}$. We will therefore test whether there is a coincidence bias in the following way. If the concentration of small particles is $C$, the concentration of coincidences is proportional to $C^{2}$. In an ice core dust sample, we would expect the ratio between small and large particles to be more or less independent of the concentration. We can therefore check for coincidence by comparing the ratio between small and large particle concentrations for different total dust concentration. In the glacial, for dust concentrations over $200 \mathrm{ppb}$, there is no correlation between the relative large particle concentration and the total concentration (figure A). For concentrations smaller than 200, the relative large particle concentration is slightly larger. This could be a climatic signal. We find no significant trend with respect to concentration, suggesting no significant coincidence effect for our measured ice core samples.

\section{Polystyrene sphere standards}

The polystyrene sphere standards were produced by BS-Partikel GmbH, Wiesbaden, Germany. $d_{\text {true }}$ is the certified diameter given by the manufacturer, $d_{\text {meas }}$ is the diameter measured by the Abakus, and $\sigma_{\text {true }}$ and $\sigma_{\text {meas }}$ are their uncertainties. 


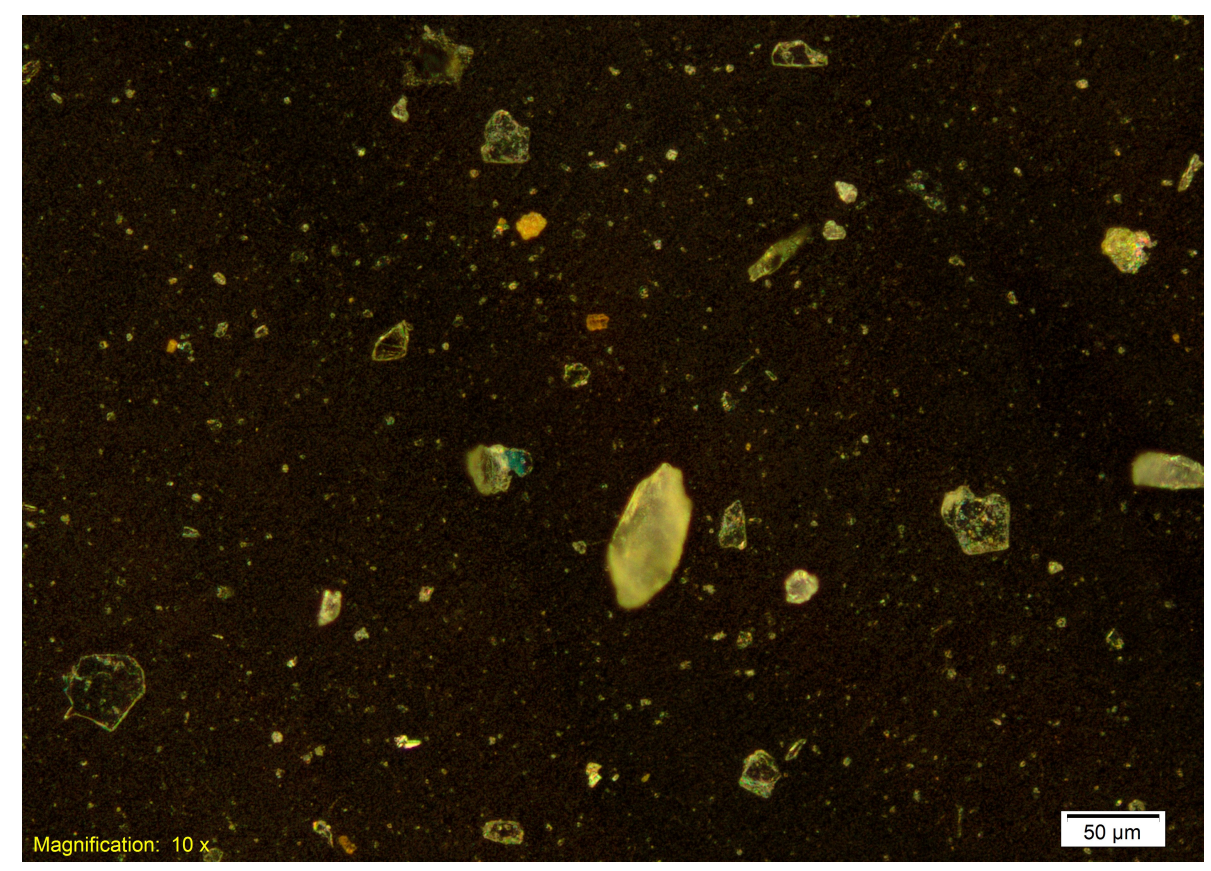

Figure B: Microscope photograph of RECAP Holocene dust from bag 837.

\begin{tabular}{rrrrrrr}
\hline $\begin{array}{r}\text { Nominal } \\
\text { diameter }(\mu \mathrm{m})\end{array}$ & $\begin{array}{r}d_{\text {true }}(\mu \mathrm{m}) \\
(\mu \mathrm{m})\end{array}$ & $\begin{array}{r}d_{\text {meas }} \\
(\mu \mathrm{m})\end{array}$ & $\begin{array}{r}\sigma_{\text {trueas }} \\
(\mu \mathrm{m})\end{array}$ & $\begin{array}{r}\text { Lotalog } \\
\text { No. }\end{array}$ \\
\hline 2 & 1.45 & 1.08 & 0.04 & 0.05 & LS0248.161 & LS0150-05 \\
4 & 1.97 & 2.06 & 0.06 & 0.05 & LS239.111 & LS0200-05 \\
5 & 4.92 & 3.12 & 0.14 & 0.27 & LS237.161 & LS0400-05 \\
10 & 10.37 & 10.83 & 0.06 & 0.35 & LS122.111 & LS0500-05 \\
\hline
\end{tabular}

\section{Microscope photographs}

Photographs of both glacial and Holocene dust were taken through a microscope (Figures B and C). The highly non-spherical shape is clearly seen. As the particles orient themselves with as low center of mass as possible on the substrate, they will typically lie on their flattest side. The aspect ratio is therefore not directly visible.

\section{E Abakus calibration scheme}

The Abakus has to be calibrated in two steps: first to yield the extinction cross section (Section 3.2), and then to account for an aspect ratio different from 1 (Section 3.5). Here is a step-by-step guide to the calibration.

1. Extinction calibration

(a) Measure standard polystyrene spheres with the Abakus, preferably at least 5 different sizes. For polar ice, the range $1-20 \mu \mathrm{m}$ is appropriate. 


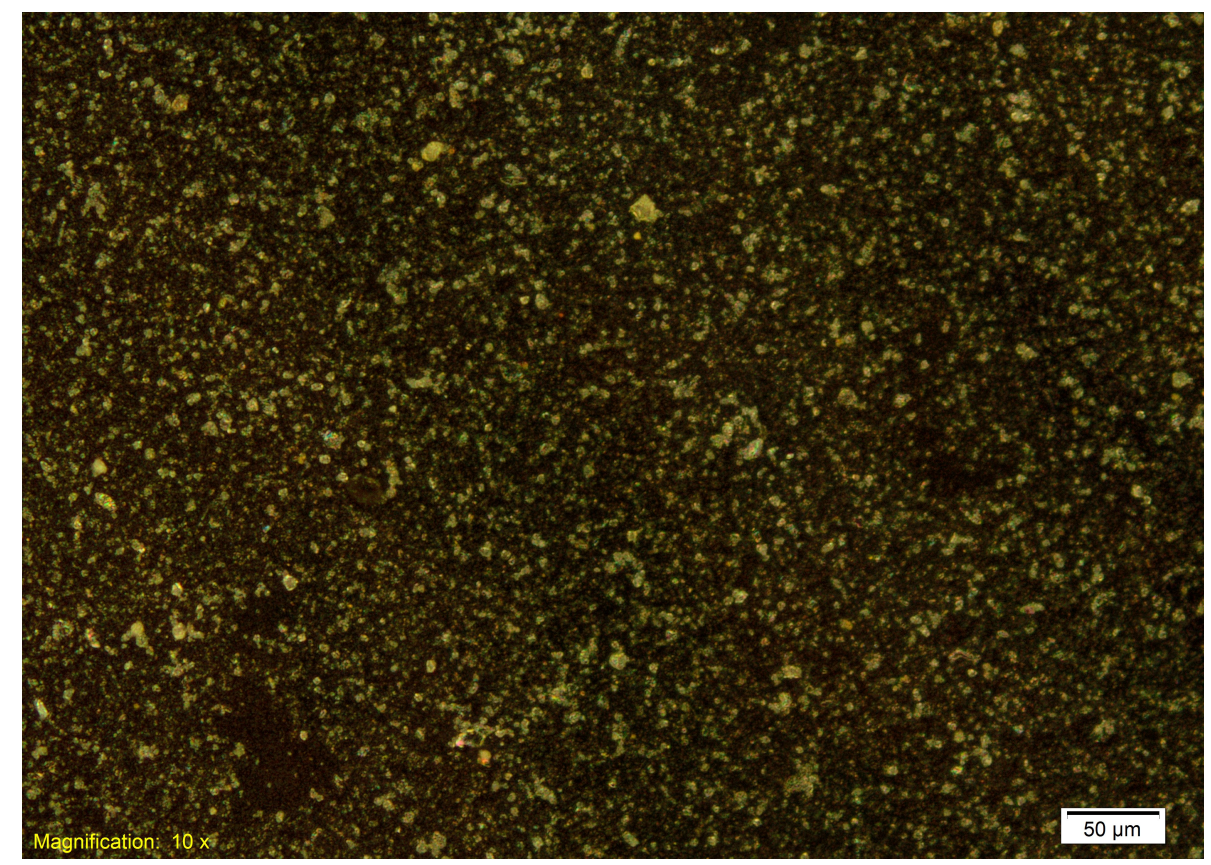

Figure C: Microscope photograph of RECAP glacial dust from bag 975.

(b) The spheres have a true diameter given by the manufacturer and now also a measured diameter given by the Abakus. The theoretical extinction diameter as a function of true diameter is shown in Figure 2. Divide the measured data by the theoretical curve to get a ratio for each data point.

(c) Connect each ratio by interpolation or preferably by fitting a smooth function to the ratio versus the measured diameter. This gives a continuous ratio function.

(d) Multiply the Abakus bin boundaries by the ratio function. The Abakus data with the new bin boundaries are now a histogram of extinction diameters.

2. Aspect ratio calibration

(a) If the aspect ratio is known, for example from SPES, multiply the Abakus bin boundaries by the cubic root of the aspect ratio. The Abakus data is now calibrated.

(b) If the aspect ratio is not known, use Coulter Counter data to calibrate the Abakus by the following optimisation algorithm. Multiply the Abakus bin boundaries by a variable $c$ to get nominally calibrated Abakus data.

(c) Take the logarithm of both Coulter Counter and Abakus data, subtract the two and take the absolute value. The sum of the absolute values is the badness of the optimisation. 


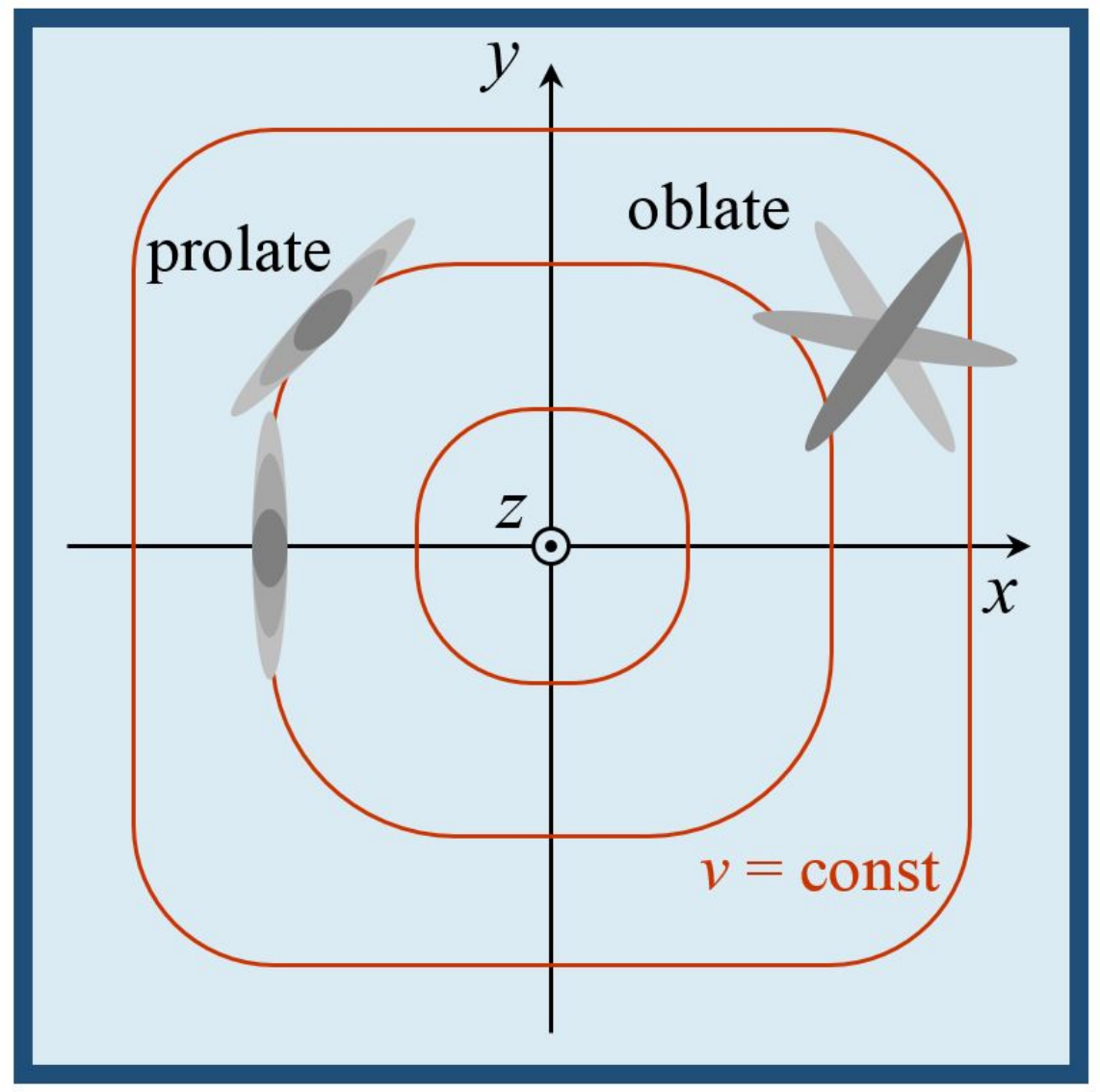

Figure D: The Abakus flow cell. The flow is in the $z$ direction and the laser beam is in the $x$ direction. The red curves are contours of constant flow velocity. Oblate particles are free to rotate in the $x, y$ plane, while prolates can rotate in a plane of constant flow.

(d) Vary $c$ to minimise badness. The nominally calibrated Abakus data corresponding to minimal badness may be used as calibrated Abakus data.

\section{F Prolate particles}

The RECAP samples consist primarily of oblate particles, so the analysis of sections 3.3 and 3.4 have not included prolate particles. The extension to prolate particles is however straight forward, and we show it here for possible future samples dominated by prolates. The aspect ratio of the RECAP samples will now be derived by comparing real Abakus data and modelled Abakus data derived from Coulter Counter data under the assumption that the particles are prolate. Prolate particles are free to rotate in a plane of constant velocity, but cannot rotate out of the plane (Jeffery, 1922). To describe their orientation, 
define a cartesian coordinate system with $z$ in the flow direction, $x$ in the laser light direction, and origin at the center of the rectangle (Figure D). If a prolate particle is located at $y=0$ and $x \neq 0$, its long side will always face the light beam, since its rotation is in the plane orthogonal to the light beam. In the $2 \mathrm{D}$ rectangle model (Figure $\mathrm{G}$ ), this corresponds to the side $a$ always facing the light. This gives a unique $d_{\text {ext }}$ for each $d_{\mathrm{vol}}$, and Equation 1 is replaced by

$$
\frac{d P\left(d_{\mathrm{ext}} \mid d_{\mathrm{vol}}\right)}{d \ln d_{\mathrm{ext}}}=\delta\left(\ln d_{\mathrm{ext}}-\ln \left(\alpha d_{\mathrm{vol}}\right)\right)
$$

where $\delta$ is the Dirac delta function and

$$
\alpha=\sqrt{\frac{\pi}{4 c}} .
$$

This value of $\alpha$ is calculated by letting $c$ be the aspect ratio of the rectangle, $d_{\text {ext }}$ the long side and $d_{\mathrm{vol}}$ the diameter of a circle with the same radius as the rectangle. In effect, Equation D means that the distribution of $d_{\text {ext }}$ is equal to the distribution of $d_{\mathrm{vol}}$, just with $d_{\mathrm{ext}}=\alpha d_{\mathrm{vol}}$. If a prolate particle is located at $x=0$ and $y \neq 0$, all rotation angles relative to the light are equally likely. It can therefore be modelled like the oblates. We assume that all positions in the $x, y$ plane are equally likely. For $x \neq 0$ and $y \neq 0, \frac{d P\left(d_{\text {ext }} \mid d_{\mathrm{vol}}\right)}{d \ln d_{\mathrm{ext}}}$ is a combination of Equation 1 and D. The combination of the equations depend on $x$ and $y$ in a non-trivial manner. However, we assume that the aspect ratio derived from the combination will lie between the aspect ratios derived from Equation 1 and D. To approximate the exact combination, we take the mean of the two distributions. Using Equation 3, modelled Abakus data can be generated from the Coulter Counter data, similar to what we did for the oblate particles. The best correspondence between modelled and real Abakus data is for $c=$ $0.34 \pm 0.04$ for the glacial and $c=0.43 \pm 0.08$ for the Holocene. As the correct relative weight of Equation 1 and D in $\frac{d P\left(d_{\text {ext }} \mid d_{\mathrm{vol}}\right)}{d \ln d_{\mathrm{ext}}}$ is uncertain, an upper bound on the aspect ratio can be calculated by using only Equation D. This gives $c=$ $0.37 \pm 0.03$ for the glacial and $c=0.45 \pm 0.06$ for the Holocene. The uncertainty on $c$ arising from a wrong relative weight of Equation 1 and $\mathrm{D}$ is therefore smaller than the uncertainty from other sources, and can be neglected. In conclusion, the aspect ratio derived from comparing Abakus to Coulter Counter data is less extreme when prolate rather than oblate particles are assumed.

ADDA simulations of prolate particles can also be used to extract an aspect ratio from the SPES data. This is rather artificial, as the SPES data shows that the particles are oblate. However, we do it to show that adding a fraction of prolates does not change the fitted aspect ratio significantly. As described in section 3.3, the mean optical thickness $\rho$ is calculated as function of extinction cross section $\sigma_{\text {ext }}$ for different aspect ratios $c$ (Figure E). These curves are interpolated to generate a contour plot of $c$ in $\rho, \sigma_{\text {ext }}$ space. The mean $\rho$ curve is then calculated for the aspect ratios $0.25,0.33$ and 0.50 for prolate particles. By fitting these mean $\rho$ curves to the oblate aspect ratio contour, it is found that they correspond to oblate particles of aspect ratio $0.23,0.28$ and 0.41 respectively. The fit is only performed for $\sigma_{\text {ext }}$ values between the 0.25 and 0.75 quantiles of the SPES data, ie. between the blue lines of Figure 6. By interpolating between the prolate/oblate aspect ratio pairs, it is found that the prolate aspect ratios corresponding to the oblate aspect ratios of 0.33 and 0.39 of 


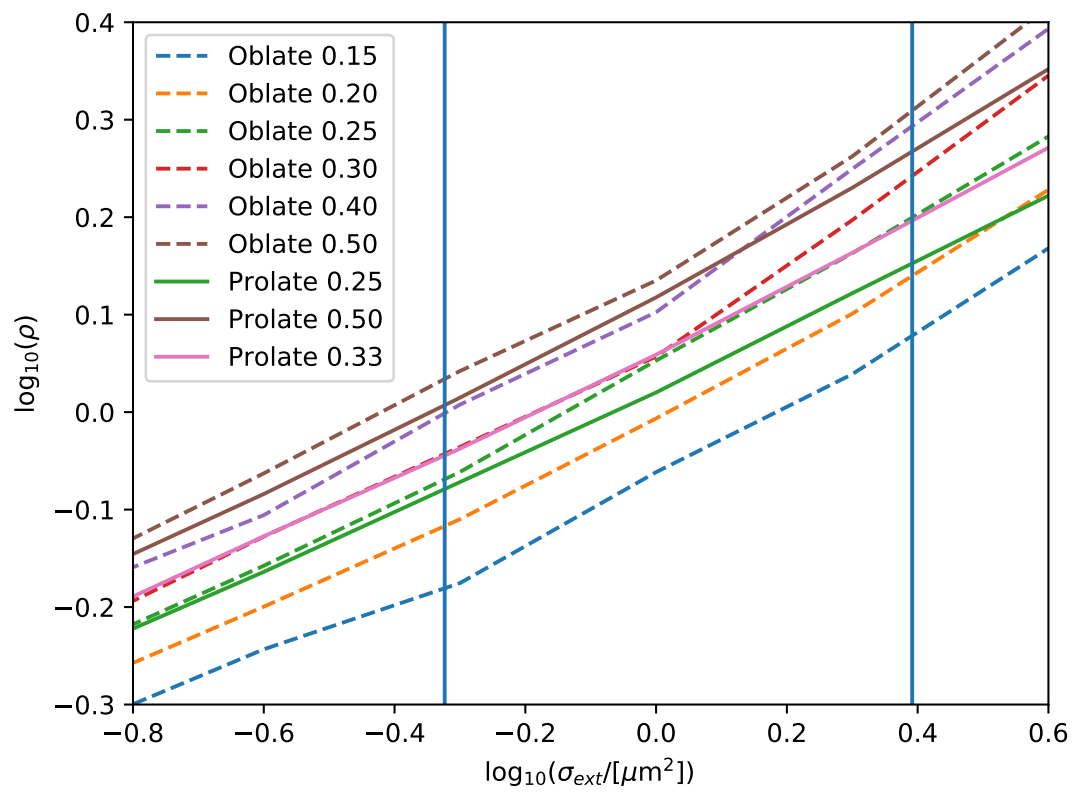

Figure E: Mean optical thickness curves for oblate and prolate particles for different aspect ratios indicated in the legend.

the RECAP glacial and Holocene samples are 0.39 and 0.47 respectively (Figure F). The ADDA simulations therefore predict less extreme aspect ratios for prolates than for oblates, similarly to the Abakus/Coulter Counter comparison. This analysis is however artificial in the sense that the RECAP samples are dominated by oblates and not prolates.

\section{G Extinction diameter calculation in the 2D model}

The particles in the Abakus are modelled as 2D rectangles, for which all rotation angles are equally likely (Figure G). The cross section of the particle is equal to $d_{\text {ext }}$ in the $2 \mathrm{D}$ model.

\section{G.1 Rod}

For calculating the cross section of a rectangle, the cross section of a rod is needed. We define a rod with length $l$ and zero width. For an angle of rotation $\phi$, the cross section is $d_{\text {ext }}=l \sin \phi$. From symmetry, the $\phi$ values are confined to $\phi \in\left[0, \frac{\pi}{2}\right]$ Assuming a uniform probability distribution for the angle, the probability of measuring the particle in the interval $[\phi, \phi+d \phi]$ is $d P(\phi ; d \phi)=$ $\frac{2}{\pi} d \phi$, which is normally written $\frac{d P(\phi)}{d \phi}=\frac{2}{\pi}$. 


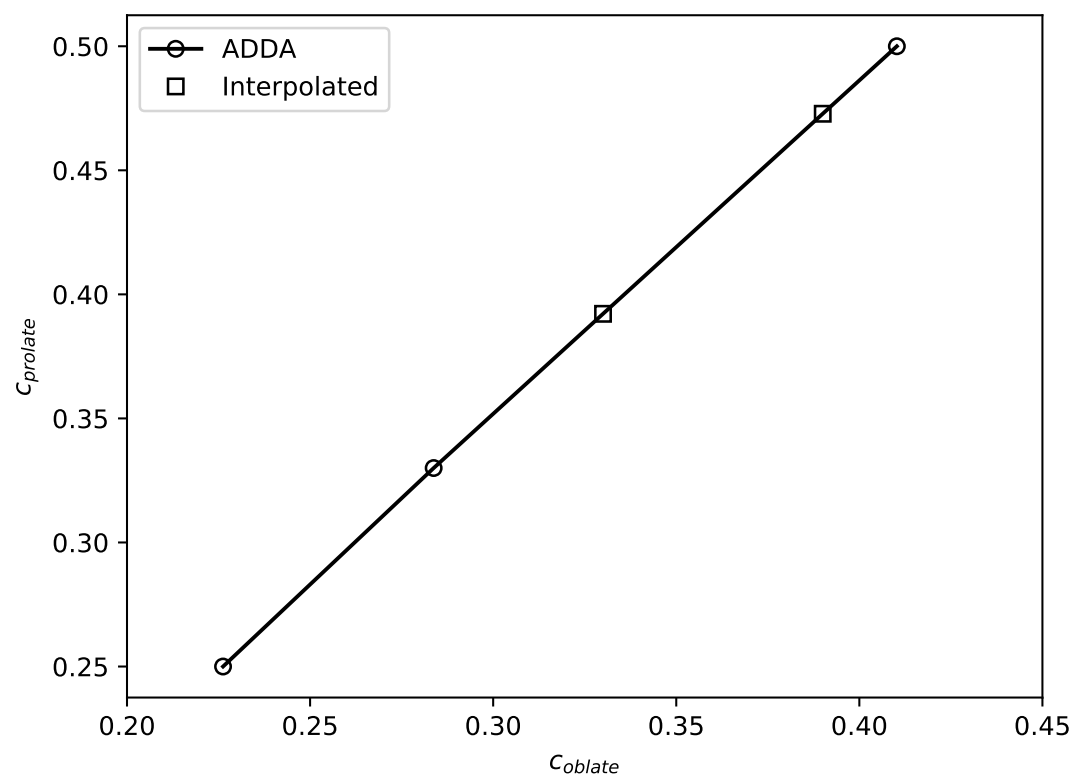

Figure F: ADDA simulated prolate aspect ratios for the corresponding oblate aspect ratios, derived from Figure E. The squares are at $c_{\text {oblate }}$ values of 0.33 and 0.39 , interpolated between the simulated values.

For converting this to $d_{\text {ext }}$ space, $d \phi$ needs to be expressed in terms of $d d_{\text {ext }}$ :

$$
\begin{aligned}
d_{\mathrm{ext}} & =l \sin \phi \\
\Rightarrow d d_{\mathrm{ext}} & =l \cos \phi d \phi \\
\Rightarrow d \phi & =\frac{1}{\sqrt{l^{2}-d_{\mathrm{ext}}^{2}}} d d_{\mathrm{ext}}
\end{aligned}
$$

Therefore

$$
\begin{aligned}
\frac{d P\left(d_{\mathrm{ext}}\right)}{d d_{\mathrm{ext}}} & =\frac{2}{\pi} \frac{1}{\sqrt{l^{2}-d_{\mathrm{ext}}^{2}}} \\
\Rightarrow \frac{d P\left(d_{\mathrm{ext}}\right)}{d \ln d_{\mathrm{ext}}} & =z
\end{aligned}
$$

where

$$
z=\frac{2}{\pi} \frac{1}{\sqrt{\left(\frac{l}{d_{\mathrm{ext}}}\right)^{2}-1}}
$$

It is seen that it diverges for $d_{\text {ext }} \rightarrow l$, because $d_{\text {ext }}$ is almost constant as a function of angle when the rod is close to being perpendicular to the light source. 


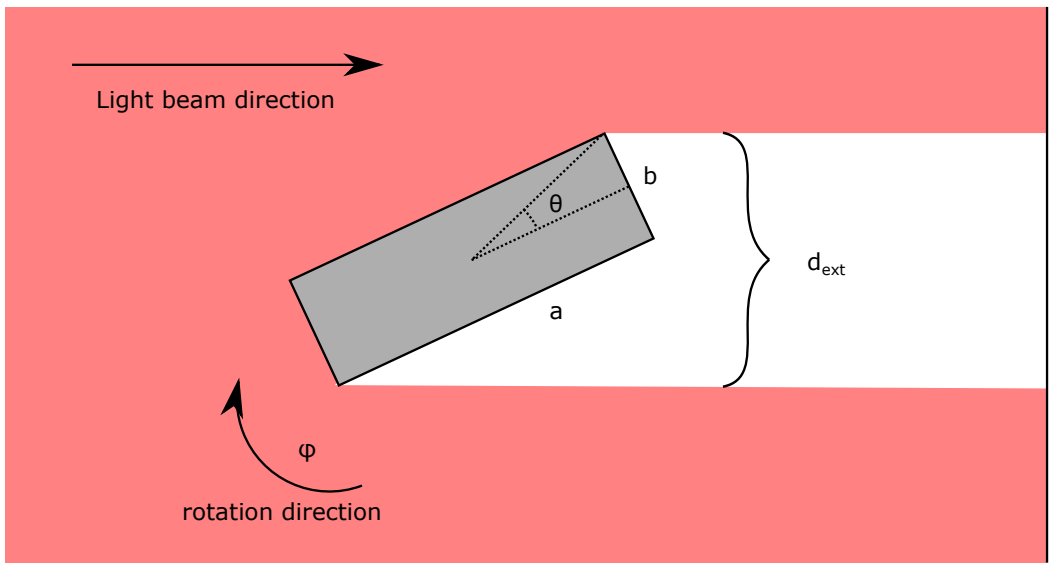

Detector

Figure G: 2D model of the particle orientation in the Abakus.

\section{G.2 Rectangle}

Consider a rectangle with side lengths $a$ and $b$. As for the rod, by symmetry, it is only necessary to consider the angles $\phi \in\left[0, \frac{\pi}{2}\right]$. In this case the cross section is given by the cross section of the diagonal. This is just the cross section of a rod rotated by

$$
\begin{aligned}
\phi_{+} & =\phi+\theta \\
\Rightarrow \phi_{+} & \in\left[\theta, \frac{\pi}{2}+\theta\right],
\end{aligned}
$$

where $\theta$ is the angle between the side $a$ and the diagonal. If $\phi$ is uniformly distributed in $\left[0, \frac{\pi}{2}\right]$, so is $\phi_{+}$in $\left[\theta, \frac{\pi}{2}+\theta\right]$. The derivation for the rod is only valid when $d_{\text {ext }}$ is a monotonous function of $\phi$. This means that it can only be directly applied for $\phi_{+}<\frac{\pi}{2}$. However, due to symmetry, the cross section is the same for $\phi_{+}=\frac{\pi}{2}+\Delta \phi$ and $\phi_{+}=\frac{\pi}{2}-\Delta \phi$, for any $\Delta \phi$. Therefore, the probability of measuring a cross section corresponding to $\phi \in\left[\frac{\pi}{2}-\theta, \frac{\pi}{2}\right]$ is twice as high as the probability of measuring the cross section of a rod in this interval. The cross section of $\frac{\pi}{2}-\theta$ is $a$. This means that the probability distribution is

$$
\frac{d P\left(d_{\mathrm{ext}}\right)}{d \ln d_{\mathrm{ext}}}= \begin{cases}0 & \text { for } d_{\mathrm{ext}}<b \\ z & \text { for } b<d_{\mathrm{ext}}<a \\ 2 z & \text { for } a<d_{\mathrm{ext}}<\sqrt{a^{2}+b^{2}}\end{cases}
$$

for

$$
z=\frac{2}{\pi} \frac{1}{\sqrt{\frac{a^{2}+b^{2}}{d_{\mathrm{ext}}^{2}}-1}} .
$$

\section{H Replicate ice core measurements}

The upper $93 \mathrm{~m}$ of the RECAP ice core has been measured twice (Figure H). This means that two parallel sticks have been cut from the core, each of which 


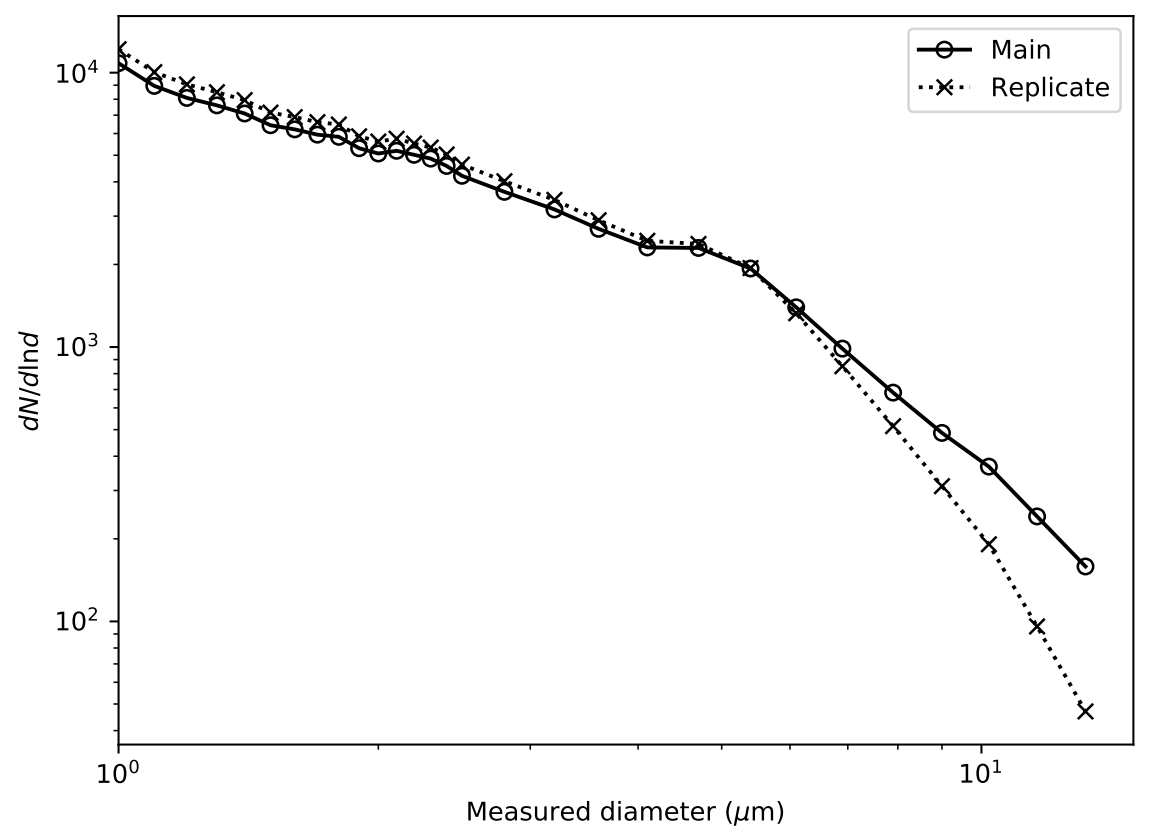

Figure H: The upper $93 \mathrm{~m}$ of the RECAP ice core measured twice with the Abakus.

has been measured on the Copenhagen CFA system. The CFA system was modified slightly between the two measurement campaigns, including changes in the pump and tubing setup, as well as general wear and roughening of the tubing walls. Therefore, the difference between the two measurements most likely reflects the error introduced by the CFA system. For the smaller particles, the replicate values are $12 \%$ larger than the main. In comparison, for the large particles the replicate measurement has up to 3 times lower values than the main measurement. This is probably because the transport of the large particles from the melt head to the instrument depends more on the specific system setup than the small particle transport.

The difference between the two measurements is used as uncertainty for the Abakus measurements. However, a mininum uncertainty of $12 \%$ is used, since the crossing of the two curves gives an artificially low differerence.

\section{Detection limit}

The number size distribution is a decreasing function even for the smallest detectable diameters (Figure I). This means that there are most likely many more particles below the detection limit than above. The total counts therefore depends heavily on where the lower cutoff is. After calibration, the Coulter Counter and the calibrated Abakus give almost the same total number of particles for the same size range. 

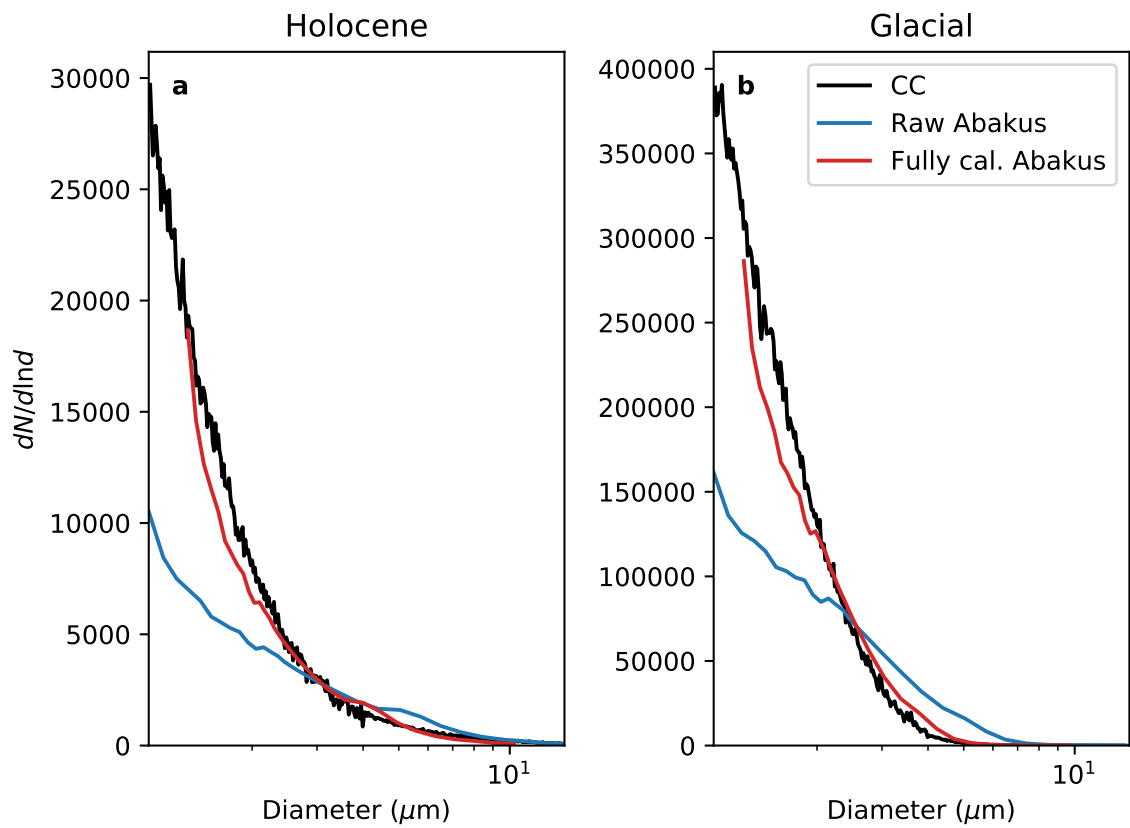

Figure I: Figure 5a,b on a linear vertical scale.

\section{References}

Jeffery, G. B.: The motion of ellipsoidal particles immersed in a viscous fluid, in: Proceedings of the royal society of London A: Mathematical, physical and engineering sciences, 715, pp. 161-179, The Royal Society, 1922.

Saey, P.: Diplomarbeit im Studiengang Physik, Master's thesis, Fakultät für Physik und Astronomie, Ruprecht-Karls-Universität Heidelberg, 1998. 